\title{
Design and Testing of a Valveless Piezoelectric Pump with Conical Vortex Diodes
}

\author{
Lei Zou, Kai Li, and Jun Huang ${ }^{*}$ \\ National Research Center of Pumps, Jiangsu University, Zhenjiang, P.R. China
}

\begin{abstract}
The valveless piezoelectric pump delivers fluid based on the difference of flow resistance of internal tube. In this paper, a vortex diode with conical tangential tube is proposed, which possesses the great characteristic of reverse cut-off, and the analysis of the forward and reverse flow resistance of the conical vortex diode is verified by finite element analysis. Then, a valveless piezoelectric pump with conical vortex diodes as the internal channel is designed, and the prototype is manufactured. The results of the output performance experiment show that the maximum output flow rate of the valveless piezoelectric pump is $10.44 \mathrm{~g} / \mathrm{min}$ at the drive frequency of $45 \mathrm{~Hz}$, and the maximum output pressure is $560 \mathrm{~Pa}$ at the drive frequency of $35 \mathrm{~Hz}$. The proposal of the valveless piezoelectric pump provides a good reference for more new types of valveless piezoelectric pumps.
\end{abstract}

\section{Introduction}

Valveless piezoelectric pumps driven by the inverse piezoelectric effect of piezoelectric materials has the advantages of simple structure, high reliability and miniaturization, which is widely applied in cell transmission, fuel cell and chip cooling.[1-3] Valveless piezoelectric pumps mostly utilize flow resistance differential tube to replace the valve. The common flow resistance differential tube is diffuser/nozzle, Tesla tube, and Y-shaped tube [4-7].

As a kind of flow resistance differential tube, the vortex diode has no mechanical moving parts and is adopted for fluid transmission. In the reverse flow of the vortex diode, there is a strong vortex motion, which exhibits a great function of reverse cut-off. Due to the characteristic of unequal flow resistances in forward and reverse, the vortex diode is utilized in the transportation of nuclear industry, chemical industry and pharmaceutical industries [8-9].

In this paper, applying the flow resistance characteristics of the diffuser/nozzle tube to the design of a vortex diode, the new vortex diodes with conical tangential tubes are proposed. And the forward and reverse flow resistances of the conical vortex diode are calculated by finite element simulation. Subsequently, a valveless piezoelectric pump with conical vortex diodes as the inner channel is designed, and the prototype is manufactured. Finally, the output performance experiment of the valveless piezoelectric pump with conical vortex diodes are carried out to verify the effectiveness of the design scheme.

\section{Working principle and simulation}

\subsection{Design and simulation}

In the previous study, the unequal flow resistance in different directions of the diffuser/nozzle had been proved, and the structure optimization of the diffuser/nozzle tube was carried out by Olssion [10]. The results demonstrate that the diffuser/nozzle tube possesses the maximum flow resistance difference with the diffusion angle is $7^{\circ}$. Moreover, the fluid in the tangential tube of the vortex diode flows in a straight line. Combining with the flow resistance characteristics of the diffuser/nozzle tube, the tangential tube of the vortex diode is structurally changed. A new vortex diode (conical vortex diodes) is designed, and the tangential tube is designed to be a diffuser and the diffusion angle is $7^{\circ}$, illustrated in Fig. 1 .

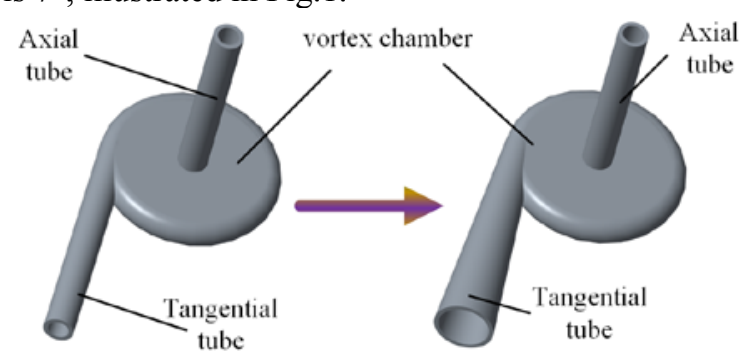

Fig. 1. Structural design of the conic vortex diode.

The process of the fluid entering the vortex chamber from the axial tube and flowing out of the tangential tube, it is defined as a forward flow. On the contrary, the process of the fluid entering the vortex chamber from the tangential tube and flowing out of the axial tube, it is defined as reverse flow. For the conical vortex diode, the resistance coefficient $\xi$ is adopted to describe the flow characteristics and defined in equations (1). The

Corresponding author: huangjun@ujs.edu.cn 
impedance ratio $\varepsilon$ is adopted to define the performance of the unidirectional transport fluid of a conic vortex diode and defined in equations (2).

$$
\begin{gathered}
\xi=\frac{2 \Delta P}{\rho v^{2}} \\
\varepsilon=\frac{\xi_{F}}{\xi_{R}}
\end{gathered}
$$

Where, $\rho\left(1000 \mathrm{~kg} / \mathrm{m}^{\wedge} 3\right)$ is the density of the fluid, $\Delta P$ $(\mathrm{Pa})$ is the pressure drops between the axial tube and the tangential tube of the conical vortex diode, and $v(\mathrm{~m} / \mathrm{s})$ is the velocity of the fluid flowing into the conical vortex diode. $\xi_{\mathrm{R}}$ is the resistance coefficient of the reverse flow, $\xi_{\mathrm{F}}$ is the resistance coefficient of the forward flow.

The fluid flows in the pump chamber in different forms during the forward and reverse flow, which results in unequal flow resistances. The characteristic of the different flow resistances can be applied to design the internal flow channels of a valveless piezoelectric pump.

\subsection{Numerical simulation}

In order to obtain the condition of fluid flowing in the conical vortex diode, the ANSYS CFX software is employed to perform the finite element analysis. The structural parameters of the conical vortex diode are shown in Table 1. The characteristics of the forward and reverse flow in conical vortex diode are evaluated by pressure drops between inlet and outlet. The flow field inside the conical vortex diode is set as the fluid domain, the material is the water. Fig. 2 is the finite element model of a conical vortex diode. In forward flow, the axial tube is defined as the inlet, the tangential tube is defined as the outlet. And in reverse flow, the tangential tube is defined as the inlet, the axial tube is defined as the outlet. The remaining wall is set as the no slip wall. The inlet boundary is determined as the pressure boundary, which is $200,500,800,1000,1500,2000$, 3000 , and $5000 \mathrm{~Pa}$. The outlet boundary is set as the open boundary (One atmosphere). In the forward flow, the fluid flowing in the conical vortex diode is not complex, and the $k-\varepsilon$ model is selected for calculation. However, in the reverse flow, there is a strong vortex motion in the vortex chamber, the RNG $k-\varepsilon$ model is selected for calculation.

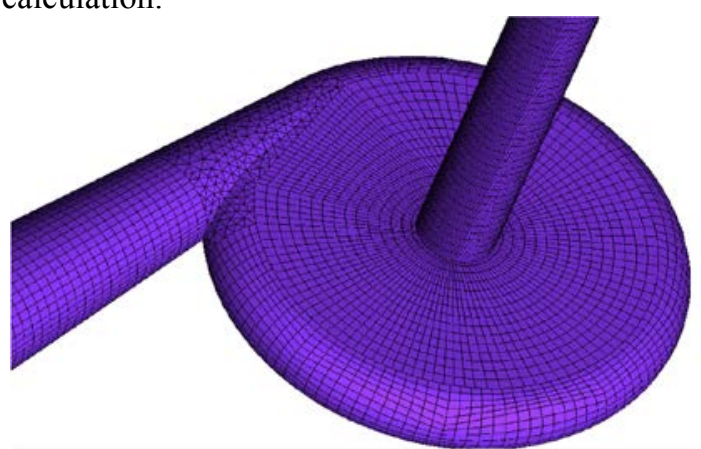

Fig.2. Finite element model of a conical vortex diode
Table 1. Structural parameters of the conical vortex diode.

\begin{tabular}{cc}
\hline Structure & Parameter \\
\hline Axial tube diameter $D \mathrm{a}$ & $1 \mathrm{~mm}$ \\
Vortex chamber height $h$ & $1 \mathrm{~mm}$ \\
Vortex chamber diameter $D_{1}$ & $6 \mathrm{~mm}$ \\
Angle of tangential tube & $7^{\circ}$ \\
\hline
\end{tabular}

Fig. 3 illustrates a velocity streamline diagram of a conical vortex diode at $500 \mathrm{~Pa}$. In the forward flow, the fluid enters the vortex chamber from the axial tube and forms a small spiral motion in the pump chamber. And then the fluid flows out from the tangential tube. In the reverse flow, the fluid enters the vortex chamber from the tangential tube, and moves along the circular wall surface. The fluid forms a vortex motion in the vortex chamber under the influence of the circular wall surface, and the vortex motion exists in the whole vortex chamber. Then the fluid flows out from the axial tube in a spiral form.

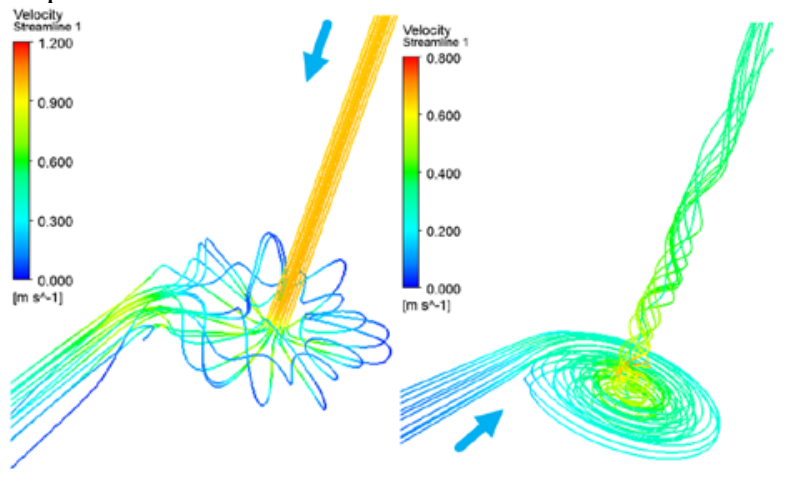

Fig.3. Streamline diagram of a conical vortex diode $(\Delta \mathrm{P}=500 \mathrm{~Pa})$

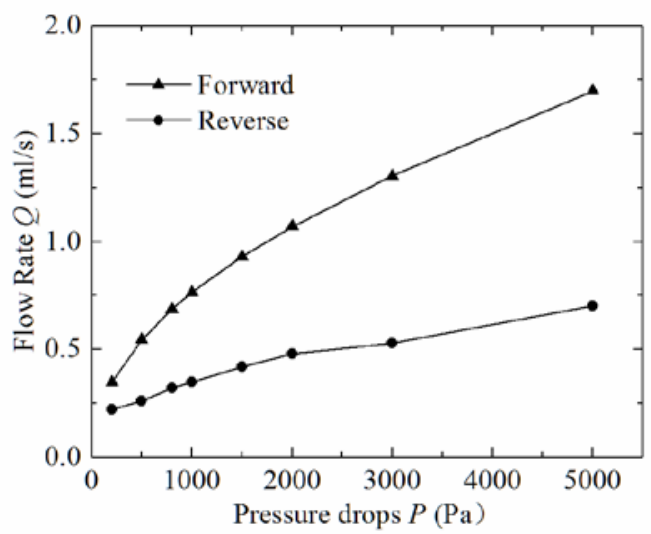

(a) Flow rate of simulation

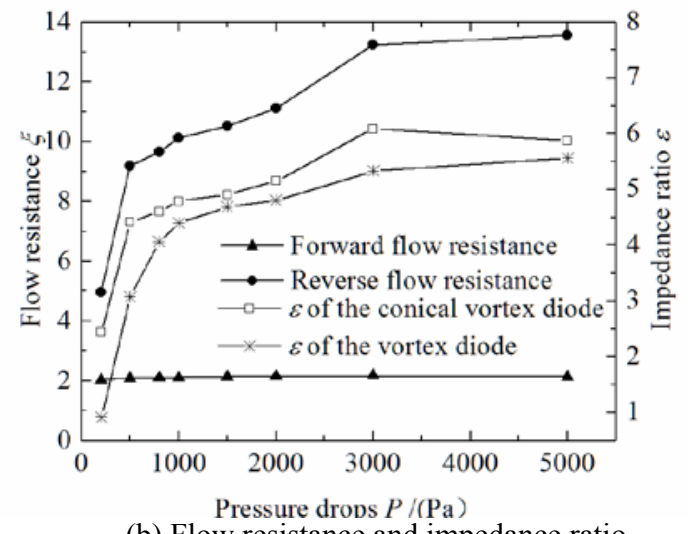

(b) Flow resistance and impedance ratio

Fig. 4. Results of simulation calculation. 
Fig. 4 illustrates the simulation results of the conical vortex diode with under various pressure drops. The flow rate in the reverse flow is always higher than that of the forward flow. Then substituting the results into equation (1), (2), the resistance coefficient $\xi$ and the impedance ratio $\varepsilon$ is obtained, shown in Fig.4 (b). As the pressure drops increases, the forward and reverse flow resistance coefficients $\xi$ increase, and the reverse flow resistance coefficient $\xi_{\mathrm{R}}$ remains greater than that of the forward flow.

Combining Fig. 3 and 4, although there is a spiral motion in the pump chamber during the forward flow, the spiral motion is small, which results in lower flow resistance. However, there is a strong vortex motion in the vortex chamber of the reverse flow, the flow resistance becomes larger. Comparing the impedance ratios $\varepsilon$ of different vortex diode under the same pressure drops, the impedance ratio $\varepsilon$ of the conical vortex diode is always higher than that of the vortex diode. The result of the comparison confirms that the design of the conical vortex diode can enhance characteristics of the unequal flow resistance. Moreover, the conical vortex diode can be applied for a valveless piezoelectric pump for oneway transmission.

\subsection{Design of the piezoelectric pump}

Fig.5 illustrates the schematic diagram of the piezoelectric pump. The piezoelectric vibrator is utilized as the power source, and the sealing ring isolates the pump chamber from the piezoelectric vibrator and keeps the pump chamber sealed, the dimensions of the seal ring is $27 \times 20 \times 0.2 \mathrm{~mm}$. A pair of conical vortex diodes are placed vertically and placed under the pump chamber, which acts as an internal "valve". The dimensions of the valveless piezoelectric pump with conical vortex diode are $40 \times 40 \times 18 \mathrm{~mm}$. The diameter of pump chamber is 20 $\mathrm{mm}$ and the depth is $1 \mathrm{~mm}$.

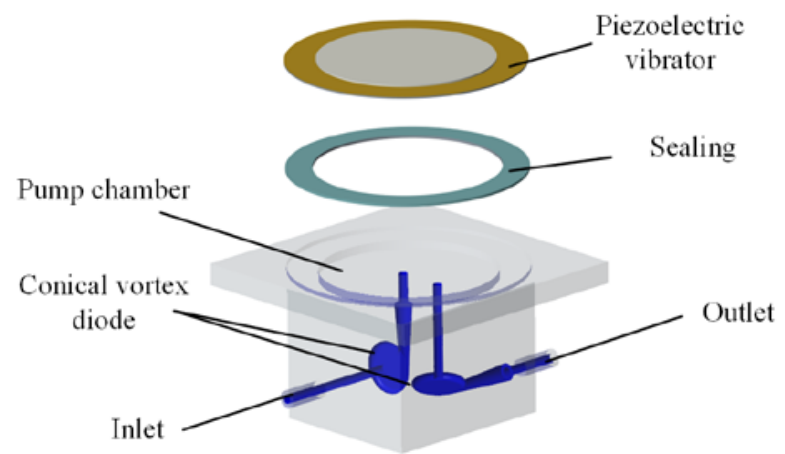

Fig.5. Schematic diagram of the piezoelectric pump

An alternating voltage is applied to the piezoelectric vibrator. Under the function of the inverse piezoelectric effect, the piezoelectric vibrator generates vibration, which causes the variation in the volume of the pump chamber. The difference in flow resistance of the conical vortex diode leads to the unequal flow under different working conditions (Up-down vibration of piezoelectric vibrator) of the piezoelectric pump. Theoretically the piezoelectric pump can realize the one-way transmission for the fluid.

\section{Experiment and results}

\subsection{Fabrication and experimention}

The prototype of the valveless piezoelectric pump with conical vortex diodes is manufactured by Stereo lithography Apparatus (SLA) technology. The processing precision of the SLA technology is $0.1 \mathrm{~mm}$, and the processing material is ABS photosensitive resin. The base material of the piezoelectric vibrator is copper alloy and the parameters of the piezoelectric vibrator are shown in Tab 2.

Fig.6 illustrates the schematic diagram of the output performance experiment. The deionized water is adopted as the fluid medium in the experiment. The signal generator provides a sinusoidal voltage, and the drive voltage is amplified by the power amplifier to $200 \mathrm{~V}$ (peak-to-peak value). An electronic balance (resolution: $0.01 \mathrm{~g}$ ) is utilized to measure the flow rate of the piezoelectric pump per minute. The output back pressure of the piezoelectric pump is evaluated by measuring the height of the fluid at the outlet tube. By changing the range of driving frequencies from $10 \mathrm{~Hz}$ to $110 \mathrm{~Hz}$, the output performance of the valveless piezoelectric pump is carried out. And the amplitude of the center position of the piezoelectric vibrator is measured by a CCD Laser displacement sensor (Keyence LK-G35, resolution: $0.001 \mathrm{~mm}$ ).

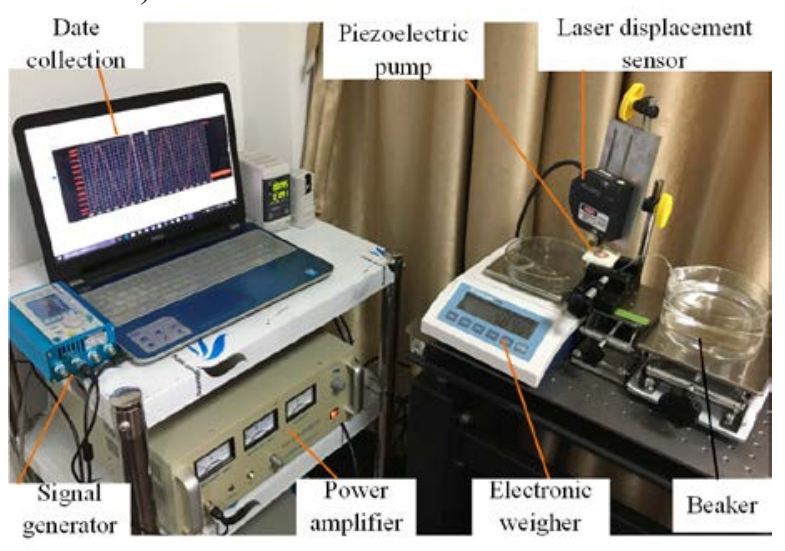

Fig. 6. Photograph of experiment.

Table 2. Structural parameters of the piezoelectric vibrator.

\begin{tabular}{cc}
\hline Structure & Parameter \\
\hline External diameter & $27 \pm 0.1 \mathrm{~mm}$ \\
Base thickness & $0.15 \mathrm{~mm}$ \\
Ceramic diameter & $19 \pm 0.2 \mathrm{~mm}$ \\
Ceramic thickness & $0.22 \mathrm{~mm}$ \\
Resonant Frequency & $4.1 \pm 0.5 \mathrm{KHz}$ \\
Resonant Impedance & $\leqslant 300 \Omega$ \\
Static Capacitance & $28000(\mathrm{PF}) \pm 30 \%$ \\
\hline
\end{tabular}

\subsection{Results and discussion}

Fig.7 (a) illustrates the output flow rate and amplitude curves varying with frequency of the valveless piezoelectric pump with conical vortex diodes. At the frequencies range of $10 \mathrm{~Hz}$ to $110 \mathrm{~Hz}$, the piezoelectric pump exhibits obvious output flow rate. The maximum 
amplitude (single peak) of the piezoelectric vibrator is $39.7 \mu \mathrm{m}$ at the drive frequency of $50 \mathrm{~Hz}$. The variation trend of the output flow rate is consistent with that of the piezoelectric vibrator. The output flow rate gradually increases with a range of $10 \mathrm{~Hz}$ to $45 \mathrm{~Hz}$, the maximum output flow rate is $10.44 \mathrm{ml} / \mathrm{min}$ at the drive frequency of $45 \mathrm{~Hz}$. Fig. 7 (b) illustrates the output back pressure and amplitude curves varying with frequencies of the valveless piezoelectric pumps with conical vortex diodes. With the change of frequencies, the vibrator amplitude curve is in the form of a parabola, and the maximum amplitude (single peak) of the piezoelectric vibrator is $44.2 \mu \mathrm{m}$ at the drive frequency of $33 \mathrm{~Hz}$. The variation of amplitude of piezoelectric vibrator affects the output back pressure of piezoelectric pump, the maximum output pressure is $560 \mathrm{~Pa}$ at the drive frequency of $35 \mathrm{~Hz}$.

The experimental results verify the reliability and effectiveness of the design scheme for the valveless piezoelectric pump with conical vortex diodes.

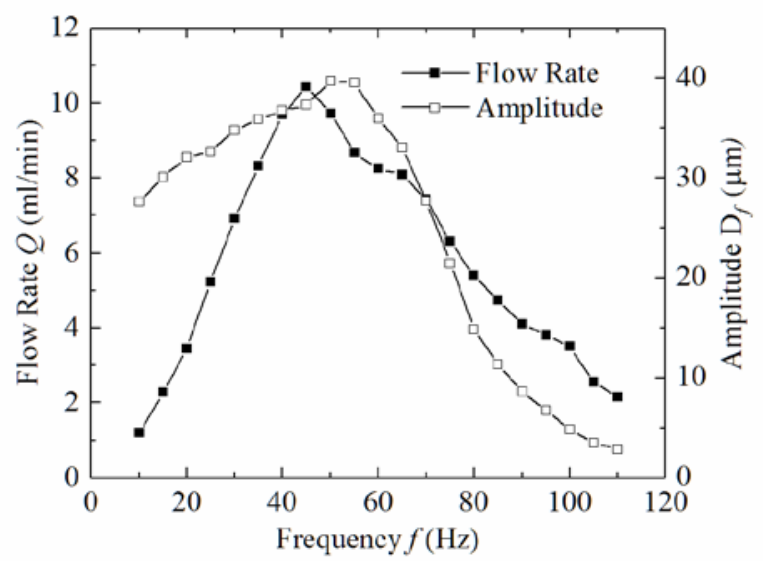

(a) Curves of output the flow rate and amplitude

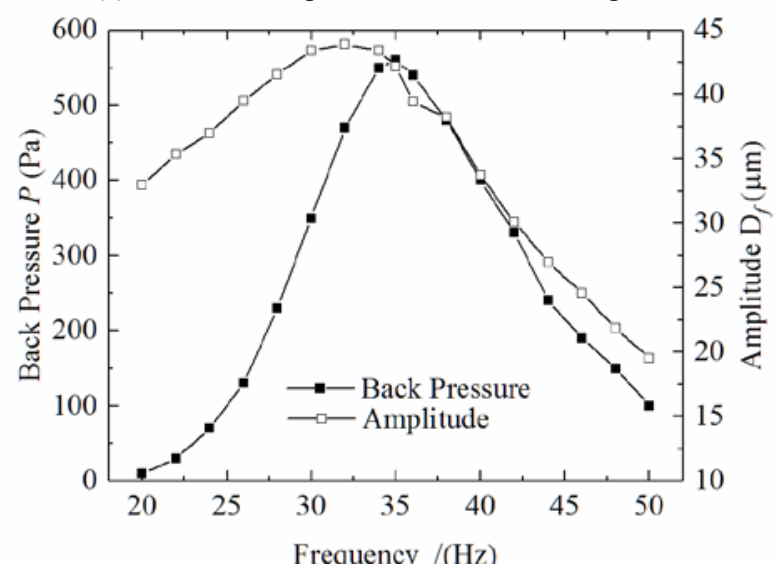

(b) Curves of the output pressure and amplitude

Fig. 7. Results of performance experimental.

\section{Conclusion}

A valveless piezoelectric pump with conical vortex diodes is designed with vortex diode as the internal flow channel. The finite element method is adopted to simulate the flow resistance of the conical vortex diode. The simulation results demonstrated that the unidirectional cut-off characteristics of the conical vortex diode. Then, a valveless piezoelectric pump with the conical vortex diode is designed, and a prototype of the valveless piezoelectric pump is fabricated. Moreover, the output performance experiment of the piezoelectric pump is carried out, and the experiment results demonstrate that the maximum flow rate is $10.44 \mathrm{ml} / \mathrm{min}$ at the drive frequency of $45 \mathrm{~Hz}$, and the maximum output pressure is $560 \mathrm{~Pa}$ at the drive frequency of $35 \mathrm{~Hz}$.

The authors would like to thank the National Natural Science Foundation of China (Grant No. 51605200) and the Senior Talent Start-up Foundation of Jiangsu University (Grant No. 14JDG145) for the financial support.

\section{References}

1. X. F. Leng, J. H. Zhang, Y. Jiang. Theory and experimental verification of spiral flow tube-type valveless piezoelectric pump with gyroscopic effect Sensors \& Actuators A Physical, 195(6):1-6 (2013).

2. J. Huang, J. H. Zhang, S. Wang. Analysis of the Flow Rate Characteristics of Valveless Piezoelectric Pump with Fractal-like Y-shape Branching Tubes Chinese Journal of Mechanical Engineering, 27(3): 628-634 (2014).

3. Q. H. Xiao, L. G. Chen, F. R. Sun. Constructal entransy dissipation rate and flow-resistance minimizations for cooling channels Science in China Series E: Technological Sciences, 53(9):2458-2468, (2010).

4. E. Stemme and G. Stemme. A valveless diffuser/nozzle based fluid pump Sensors \& Actuators A 39 :159-167 (1993)

5. R. Gamboa, C. J. Morris, F. K. Forster. Improvement Fixed Valve Micropump Performance Through Shape Optimization of Valves Journal of Fluids Engineering, 127(2):339, (2005).

6. J. Huang, J. Zhang, W. Shi. 3D FEM analyses on flow field characteristics of the valveless piezoelectric pump. Chinese Journal of Mechanical Engineering, 29(4):825-831 (2016)

7. S. S. Wang, X. Y. Huang, C, Yang. Valveless micropump with acoustically featured pumping chamber Microfluidics \& Nanofluidics, 8(4):549555 (2010).

8. S. X. Qu, Y. H. Wu, Z, Z He. Surrogate Fluid Experimental Study and CFD Simulation on the Hydraulic Characteristics of Vortex Diode Nuclear Science and Engineering, 1-8, (2017).

9. Jain P, Bhandari V M, Balapure K. Hydrodynamic cavitation using vortex diode: An efficient approach for elimination of pathogenic bacteria from water Journal of environmental management, 242: 210219, (2019).

10. Olsson A. Olsson A. Valveless diffuser micropumps Signaler, sensorer och system, (1998). 